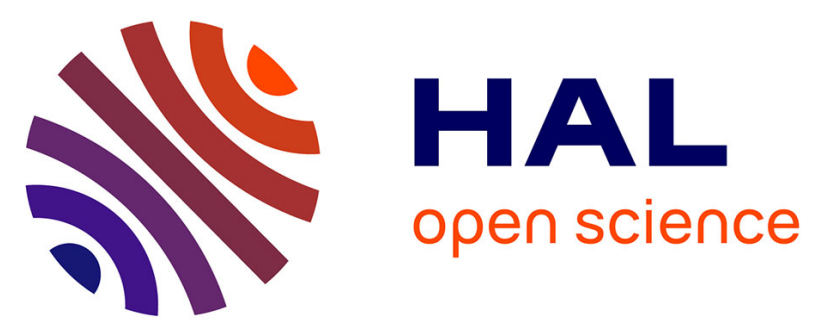

\title{
Association of Self-reported Diabetes with Age-related Macular Degeneration in the EUREYE study
}

Fotis Topouzis, Eleftherios Anastasopoulos, Cristina A Augood, Graham C

Bentham, Usha Chakravarthy, Paulus T V M de Jong, Mati Rahu, Johan

Seland, Gisele Soubrane, Laura Tomazzoli, et al.

\section{To cite this version:}

Fotis Topouzis, Eleftherios Anastasopoulos, Cristina A Augood, Graham C Bentham, Usha Chakravarthy, et al.. Association of Self-reported Diabetes with Age-related Macular Degeneration in the EUREYE study. British Journal of Ophthalmology, 2009, 93 (8), pp.1037-n/a. 10.1136/bjo.2008.146316 . hal-00477813

\section{HAL Id: hal-00477813 https://hal.science/hal-00477813}

Submitted on 30 Apr 2010

HAL is a multi-disciplinary open access archive for the deposit and dissemination of scientific research documents, whether they are published or not. The documents may come from teaching and research institutions in France or abroad, or from public or private research centers.
L'archive ouverte pluridisciplinaire HAL, est destinée au dépôt et à la diffusion de documents scientifiques de niveau recherche, publiés ou non, émanant des établissements d'enseignement et de recherche français ou étrangers, des laboratoires publics ou privés. 


\section{Association of Diabetes with Age-related Macular Degeneration in the EUREYE study}

Topouzis F, ${ }^{1}$ Anastasopoulos E, ${ }^{1}$ Augood C, ${ }^{2}$ Bentham GC, ${ }^{3}$ Chakravarthy $\mathrm{U},{ }^{4}$ de Jong PTVM, ${ }^{5}$ Rahu M, ${ }^{6}$ Seland J, ${ }^{7}$ Soubrane G, ${ }^{8}$ Tomazzoli L, ${ }^{9}$ Vingerling JR, ${ }^{5}$ Vioque J, ${ }^{10}$ Young IS, ${ }^{11}$ Fletcher $\mathrm{AE}^{2}$

${ }^{1}$ Department of Ophthalmology, Aristotle University of Thessaloniki School of Medicine, Greece

${ }^{2}$ Department of Epidemiology \& Population Health, London School of Hygiene \& Tropical Medicine UK

${ }^{3}$ Centre for Environmental Risk, University of East Anglia, UK

${ }^{4}$ Department of Ophthalmogy, Queen's University of Belfast, UK

${ }^{5}$ The Netherlands Ophthalmic Research Institute, KNAW, and Department of Ophthalmology, Academic Medical Centre Amsterdam, The Netherlands

${ }^{6}$ Department of Epidemiology and Biostatistics, National Institute for Health Development, Tallinn, Estonia

${ }^{7}$ Øyeavdelingen, Haukeland Sykehus University of Bergen, Norway

${ }^{8}$ Clinique Ophthalmologique, Universitaire De Creteil, Paris, France

${ }^{9}$ Clinica Oculistica, Università degli Studi di Verona, Italy

${ }^{10}$ Dpto. Salud Publica Universidad Miguel Hernández, Alicante, and CIBERESP, Ciber de Epidemiología y Salud Pública, Spain

${ }^{11}$ Centre for Clinical and Population Sciences, Queen's University of Belfast, UK

\section{Corresponding author}

Fotis Topouzis, MD

Aristotle University of Thessaloniki

AXEPA Hospital

S. Kiriakidi 154640

Thessaloniki, Greece

Tel. +302310994920

e-mail: ftopouzis@otenet.gr

Competing Interest: None declared

Running head: Association of diabetes mellitus with AMD in EUREYE study

Ethics Approval: Ethics approval was obtained at each center from the relevant ethics committee. Study participants gave informed written consent prior to participation and blood sample drawing.

Keywords: diabetes, age-related macular degeneration, Eureye study 
Financial support: European Commission Vth Framework, Brussels, Belgium (contract no. QLK6-CT-1999-02094). Additional funding for cameras was provided by the Macular Disease Society, Andover, United Kingdom. Prof Rahu was financed by the Ministry of Education and Science, Tallinn, Estonia (target funding no. 01921112s02). Additional funding in Alicante was received from the Spanish Ministry of Health (Grants: FIS 01/1692E; CIBERESP). European Eye investigator meetings were supported by travel grants from Novartis, Basel, Switzerland, and Pfizer Inc., New York, New York.

Licence for publication: the Corresponding Author has the right to grant on behalf of all authors and does grant on behalf of all authors, an exclusive licence (or non-exclusive for government employees) on a worldwide basis to the BMJ Publishing Group Ltd and its Licensees to permit this article (if accepted) to be published in BJO and any other BMJPGL products to exploit all subsidiary rights, as set out in our licence . 


\begin{abstract}
Objective: To examine the association between self-reported diabetes history and early or late age-related macular degeneration (AMD) in the European population.

Methods: Participants aged 65 years and over in the cross-sectional population- based EUREYE study underwent an eye examination including digital retinal photography. The images were graded at a single center. A structured questionnaire was administered by trained fieldworkers for putative risk factors for AMD including history of diabetes mellitus. Logistic regression models were used to examine the association between diabetes and stages of AMD, taking account of potential demographic, behavioral, dietary, and medical (history of cardiovascular disease) confounders.
\end{abstract}

Main Outcome Measures: Photographic images were graded according to the modified International Classification System for AMD and stratified into 5 exclusive stages from no signs of AMD (AMD stage 0), early AMD (Stages 1-3) and late AMD (Stage 4). Late AMD was subdivided in neovascular AMD (NV-AMD) or geographic atrophy (GA).

Results: Data on diabetes history and potential confounders were available in 2117 controls subjects without AMD, 2182 with early AMD, 49 with GA, and 101 with NV-AMD. Of all participants, $13.1 \%$ reported a history of diabetes. After adjusting for potential confounders, subjects with neovascular AMD compared to controls had increased odds for diabetes (odds ratio 1.81; $95 \%$ confidence interval, 1.10-2.98, $\mathrm{p}=0.02$ ). Subjects with AMD grades 1-3 or GA had no increased odds for diabetes compared to those without AMD.

Conclusions: In the EUREYE study, after multiple adjustments, positive association of diabetes mellitus with neovascular AMD was found. The hypothesis that diabetes is associated with neovascular AMD but not with geographic atrophy may suggest different pathogenesis of the two advanced forms of the disease and needs to be further evaluated. 


\section{Introduction}

Age-related macular degeneration (AMD) is an important cause of irreversible visual impairment or blindness in the adult Western World. ${ }^{1}$ The prevalence of AMD and the subsequent burden in public health are expected to rise as a result of the progressive increase in the life expectancy and the proportion of elderly persons in the population. The identification and modification of risk factors may have the potential for improving knowledge of pathogenetic mechanisms, facilitating prevention and subsequently reducing the morbidity associated with the disease.

The pathogenesis of AMD is considered to be multifactorial. Although many previous studies assessed a considerable number of demographic, environmental, genetic or medical risk factors for AMD, the majority remains controversial. AMD and cardiovascular disease have been hypothesized to share common pathways. ${ }^{2,3}$ Diabetes mellitus as a major cause of cardiovascular disease has been linked also with AMD. However, to date most of the epidemiological data have not been consistent regarding the association of diabetes with AMD with some studies finding an association, ${ }^{3-7}$ others not ${ }^{8-14}$, while one study reported an inverse relationship between diabetes and incident reticular drusen. ${ }^{15}$ In addition, even in studies that found an association of diabetes with AMD, the specific association with types of late AMD (neovascular AMD or geographic atrophy) was also inconsistent.

The EUREYE study is a multi-center, population-based, analytic cross sectional study of risk factors for AMD with retrospective and current exposure measurements. ${ }^{16}$ The study centers (Estonia, France, Greece, Italy, Norway, Spain, UK) were chosen to maximize the range of geographical latitude and lifestyle behaviors, including diet since primary hypotheses were related to sunlight exposure and antioxidants. We examined the association of diabetes with AMD stages and late AMD types in the EUREYE study.

\section{Materials and Methods}

\section{Population}


The methods used to identify and describe this population have been published previously. ${ }^{16}$ In brief, participants were recruited from random sampling of the population aged over 65 years and attended the examination centre where they were first interviewed by trained fieldworkers and then underwent an ophthalmological examination and gave a non-fasting blood sample.

\section{Risk factors assessment}

Measurement of general lifestyle and medical history risk factors: Sociodemographic details and educational level were recorded. Self-reported data on current and past smoking, alcohol consumption habits, history of cardiovascular disease (stroke or heart attack), angina, and use of aspirin were collected. A history of diabetes mellitus was defined as a positive answer to the question: "Have you ever been told by a doctor that you have diabetes (sugar in the blood)?" For each study centre the years of education were classified into tertiles: low education group (for the lower third), middle (the middle third) and high (upper third).

\section{Measurement of other risk factors:}

We measured weight and demispan (the distance between the sternal notch and the finger roots with the arm out-stretched laterally). The demiquet index was calculated as weight /demispan ${ }^{2}$ For comparison with other studies we also calculated Body Mass Index as weight $(\mathrm{kg}) / \mathrm{height}^{2}$ (in meters) in which height was calculated from demispan using equations derived from a sub study in the Alicante population. ${ }^{17}$ Systolic and diastolic blood pressures were measured with the arm in the seated position after 3 to 5 minutes rest. Two readings were taken with a 5minutes interval and the average of the two values recorded was used. Fieldworkers in all centers followed the same protocol and used the same type of sphygmomanometer (OMRON HEM 705 $\mathrm{CP})$.

\section{Fundus photography and grading}

Following pupillary dilation with tropicamide $0.5 \%$ and phenylephrine $5 \%$, two $35^{\circ}$ nonsimultaneous stereoscopic colour fundus images were taken of each eye, centred on the fovea. Images from participants were saved without manipulation as raw TIFF files to compact discs and sent to the grading centre in Rotterdam. The fundus images were graded according to a modification of the International Classification and Grading System for AMD. ${ }^{18}$ In this system, all AMD fundus signs within a standard circle (diameter $6 \mathrm{~mm}$ ) around the fovea are recorded. Two highly experienced staff in the Rotterdam Fundus Photography Reading Centre undertook 
grading of the fundus. We next categorized eyes into five mutually exclusive stages from 0 to 4 according to fundus signs that had an increasing risk of late AMD. No AMD was defined as stage 0 , meaning no signs of AMD at all or only hard drusen $(<63 \mu \mathrm{m})$. Stage 1 was defined as soft distinct drusen $(\geq 63 \mu \mathrm{m})$ or pigmentary abnormalities, stage 2 as soft indistinct drusen $(\geq$ $125 \mu \mathrm{m})$ or reticular drusen only or soft distinct drusen $(\geq 63 \mu \mathrm{m})$ with pigmentary abnormalities, and stage 3 as soft indistinct drusen $(\geq 125 \mu \mathrm{m})$ or reticular drusen with pigmentary abnormalities. Stage 4 was similar to late AMD subdivided into geographic atrophy or neovascular AMD. Geographic atrophy (GA) was defined as any sharply demarcated round or oval area of apparent absence of the RPE, larger than $175 \mu \mathrm{m}$, with visible choroidal vessels, and no wet AMD. Neovascular (NV) AMD was defined as the presence of a serous or hemorrhagic detachment of the RPE and/or a sub retinal neovascular membrane and/or sub retinal haemorrhage, and/or periretinal fibrous scarring, even with patches of GA. ${ }^{19}$ In the total sample size of 4753 participants with gradable fundus images, there were 2262 controls persons with grade 0, 2333 early AMD (1734 grade 1, 482 grade 2, 117 grade 3), and 158 late AMD (109 cases of NV-AMD, and 49 cases of GA). ${ }^{20}$

Fundus images were also graded for the presence of retinal haemorrhages. Retinal haemorrhages were graded as present if lesions with the characteristics of blood (deep red or reddish brown confluent areas with dot or blot configuration) were seen in the neurosensory retina. Retinal haemorrhages were categorized as unilateral or bilateral depending on their presence in one or both eyes of an individual.

\section{Blood samples}

Blood samples packed in dry ice were shipped to the study's central laboratory (Queen's University, Belfast) on a monthly basis and stored at $-70^{\circ} \mathrm{C}$ until analysis by reverse phase HPLC for lutein, zeaxanthein, and alpha tocopherol. Total ascorbate was measured using an enzymebased assay in plasma stabilized with metaphosphoric acid. Cholesterol was measured using an enzymatic assay (Randox, Crumlin, UK) on a Cobas FARA centrifugal analyser (Roche Diagnostics, UK).

\section{Statistical analysis}

Statistical analysis was carried out using STATA (release 9.0, Stata Corp., College Station, TX). We took account of the study design (seven centres) by estimating robust standard errors and 
corresponding p values and $95 \%$ confidence intervals using the survey suite of commands in STATA.

Logistic regression was used to examine associations between history of diabetes and each stage of AMD and the two types of late AMD. In all analyses the control group had no AMD. Possible confounding variables included age, sex, smoking (current, ex and never), level of education, body mass index (BMI), alcohol consumption, history of cardiovascular disease or angina, systolic blood pressure, aspirin use, and serum alpha-tocopherol to cholesterol ratio, vitamin $\mathrm{C}$ and lutein.

Self-reported diabetes history was available in 4722 participants and complete data on confounders in 4247.

\section{Results}

Overall the prevalence of self -reported diabetes was $13.1 \%(n=616)$. Compared to those without diabetes, people with diabetes were more likely to be male, to have a lower level of education, to report a positive history of cardiovascular disease or angina, to report a regular use of aspirin, to have higher BMI, to have a higher systolic blood pressure (SBP), to have lower blood level of total cholesterol, lower level of Vitamin C and higher alpha-tocopherol to cholesterol ratio (Table 1).

Table 1: Characteristics of people with and without a history of diabetes in the EUREYE Study ( $n=4,247$ with data on full confounders)

\begin{tabular}{|c|c|c|c|}
\hline & $\begin{array}{c}\text { Diabetes } \\
(\mathrm{n}=561)\end{array}$ & $\begin{array}{c}\text { No Diabetes } \\
(\mathrm{n}=3686)\end{array}$ & P-value \\
\hline Age $^{1}$ (years) & & $73.09(5.67)$ & 0.3 \\
\hline Sex (n,\%men) & $72.86(5.00)$ & $1623(44.0)$ & 0.04 \\
\hline Lowest tertile of education (n,\%) & $284(50.6)$ & & \multirow{2}{*}{0.02} \\
\hline & $229(40.8)$ & $1370(37.2)$ & \\
\hline Smoking (n,\%) & & & \multirow{2}{*}{0.4} \\
\hline - Never & $289(51.5)$ & $1965(53.3)$ & \\
\hline - Ex & $199(35.5)$ & $1188(32.2)$ & \\
\hline - Current & $73(13.0)$ & $533(14.5)$ & \\
\hline
\end{tabular}




\begin{tabular}{|c|c|c|c|}
\hline - Never & $114(20.3)$ & $567(15.4)$ & \multirow{3}{*}{0.08} \\
\hline - Infrequent & $223(39.8)$ & $1618(43.9)$ & \\
\hline - weekly or more & $224(39.9)$ & $1501(40.7)$ & \\
\hline Cardiovascular disease $^{2}(\mathrm{n}, \%)$ & $124(22.1)$ & $461(12.5)$ & 0.0001 \\
\hline Angina $^{3}(n, \%)$ & $143(25.5)$ & $450(12.2)$ & 0.001 \\
\hline Retinal haemorrhages either eye & $102(18.6)$ & $162(4.5)$ & $<0.00001$ \\
\hline Regular use of aspirin ${ }^{5}(n, \%)$ & $143(25.5)$ & $739(20.1)$ & 0.06 \\
\hline $\mathrm{BMI} \geq 30(\mathrm{n}, \%)$ & $256(45.6)$ & $1273(34.5)$ & 0.002 \\
\hline $\mathrm{SBP}^{1}, \mathrm{mmHg}$ & $155.38(23.40)$ & $149.88(22.34)$ & 0.03 \\
\hline Total cholesterol $^{\prime}, \mu \mathrm{mol} / \mathrm{l}$ & $5.35(1.12)$ & $5.77(1.11)$ & 0.02 \\
\hline Vitamin $C^{6}, \mu \mathrm{mol} / \mathrm{l}$ & $40.0(22.6-56.4)$ & $45.4(27.5-62.4)$ & 0.02 \\
\hline Lutein $^{6}(\mu \mathrm{mol} / \mathrm{l})$ & $0.123(0.049-0.286)$ & $0.113(0.047-0.276)$ & 0.7 \\
\hline Zeaxanthin $^{6}(\mu \mathrm{mol} / \mathrm{l})$ & $0.029(0.016-0.057)$ & $0.028(0.015-0.054)$ & 0.7 \\
\hline $\begin{array}{l}\text { Alpha- tocopherol }{ }^{6, I}(\mu \mathrm{mol} / \mathrm{mmol} \\
\text { cholesterol) }\end{array}$ & $5.55(4.85-6.47)$ & $5.19(4.49-5.94)$ & 0.003 \\
\hline & & & \\
\hline
\end{tabular}

${ }^{1}$ Mean (standard deviation)

${ }^{2}$ history of heart attack or stroke

${ }^{3}$ information missing in 7 people ( 1 with diabetes and 6 without diabetes)

${ }^{4}$ information missing in 92 people (11 with diabetes and 81 without diabetes)

${ }^{5}$ at least once a week

${ }^{6}$ Median (interquartile range)

${ }^{7}$ Ratio of alpha tocopherol to cholesterol

Diabetes was strongly associated with the presence of retinal haemorrhages as identified by the fundus images grading (Table 1). In analyses adjusted for age, sex, systolic pressure and history of cardiovascular disease, people with diabetes were significantly more likely to have retinal haemorrhages OR: $4.37,95 \%$ CI:3.77- 5.08, $\mathrm{p}<0.0001$. The association of diabetes with bilateral retinal hemorrhages was even stronger; the adjusted OR for diabetes was $22.64,95 \%$ CI: 14.89-34.46, $\mathrm{p}<0.0001$.

The prevalence of diabetes among the five AMD groups is presented in Table 2. 
Table 2. Prevalence of self-reported diabetes by AMD grade in the EUREYE study. $(n=4722,31$ subjects with missing data on diabetes)

\begin{tabular}{|c|c|c|c|}
\hline AMD & $\begin{array}{c}\text { No Diabetes } \\
\mathrm{n}(\%)\end{array}$ & $\begin{array}{c}\text { Diabetes } \\
\mathrm{n}(\%)\end{array}$ & Total \\
\hline No AMD & $1,954(87.0)$ & $292(13.0)$ & 2,246 \\
\hline AMD grade 1 & $1,491(86.6)$ & $231(13.4)$ & 1,722 \\
\hline AMD grade 2 & $430(89.6)$ & $50(10.4)$ & 480 \\
\hline AMD grade 3 & $100(86.2)$ & $16(13.8)$ & 116 \\
\hline Late AMD & $131(82.9)$ & $27(17.1)$ & 4722 \\
\hline Total & $4,106(87.0)$ & $616(13.1)$ & 158 \\
\hline
\end{tabular}

The prevalence of diabetes among subjects with GA was $13.2 \%$, while the prevalence of diabetes among subjects with neovascular AMD was 19.3\%. In the regression analysis, the association of diabetes with grades of AMD was adjusted for age and sex. Diabetes was not associated with early AMD grades nor with geographic atrophy. In contrary, subjects with neovascular AMD were 1.75 times more likely to have diabetes $(\mathrm{p}=0.016)$ compared to those with AMD grade 0 (controls). The results were additionally adjusted for the following parameters: smoking, educational level, alcohol intake, body mass index (BMI), self-reported history of cardiovascular disease, regular aspirin intake, systolic blood pressure, serum alphatocopherol to cholesterol ratio, vitamin $\mathrm{C}$ and lutein level; the association did not change significance. After multiple adjustments, subjects with neovascular AMD were 1.81 times more likely to have diabetes $(\mathrm{p}=0.02$ ), compared to those without AMD. Either AMD grades 1-3 or GA were not associated with diabetes in the fully adjusted model. (Table 3)

Table 3. Association between diabetes mellitus and AMD by grade and type of AMD in the EUREYE study.

\begin{tabular}{|l|l|l|}
\hline & $\mathrm{OR}^{*}(95 \% \mathrm{CI})^{\dagger}$ & $\mathrm{OR}^{*}(95 \% \mathrm{CI})^{*}$ \\
\hline AMD grade 1 & 1.03 & 1.04 \\
$\mathrm{n}=1552$ & $0.77-1.38$ & $0.76-1.40$ \\
\hline
\end{tabular}




\begin{tabular}{|l|l|l|}
\hline & $\mathrm{p}=0.8$ & $\mathrm{p}=0.8$ \\
\hline AMD grade 2 & 0.75 & 0.75 \\
$\mathrm{n}=430$ & $0.54-1.06$ & $0.54-1.04$ \\
& $\mathrm{p}=0.1$ & $\mathrm{p}=0.1$ \\
\hline AMD grade 3 & 1.09 & 1.14 \\
$\mathrm{n}=108$ & $0.69-1.75$ & $0.77-1.71$ \\
& $\mathrm{p}=0.7$ & $\mathrm{p}=0.5$ \\
\hline Geographic atrophy (GA) & 1.08 & 1.07 \\
$\mathrm{n}=38$ & $0.31-3.70$ & $0.28-4.04$ \\
& $\mathrm{p}=0.9$ & $\mathrm{p}=0.9$ \\
\hline Neovascular AMD & 1.75 & 1.81 \\
$\mathrm{n}=95$ & $1.11-2.76$ & $1.10-2.98$ \\
& $\mathbf{p}=\mathbf{0 . 0 1 6}$ & $\mathbf{p}=\mathbf{0 . 0 2}$ \\
\hline
\end{tabular}

* in all analyses the comparison group are those with ARM grade $0, n=2024$ with data on full confounders

$\dagger$ adjusted for age and sex

$\$$ adjusted for age,sex, smoking, education, BMI, alcohol consumption, cardiovascular disease, aspirin use, systolic blood pressure, alpha-tocopherol ratio, vitamin C, lutein

\section{Discussion}

In the EUREYE Study, an association between self-reported diabetes mellitus and neovascular AMD was observed. This association remained significant after adjusting for potential confounders. No association was found of diabetes history with any stage of early AMD (stages 1-3) or GA.

Diabetes related changes in the function and structure of the retinal pigment epithelium, Bruch's membrane, and the choroidal circulation have been hypothesized to increase the risk of AMD. Histopathologic studies in eyes of subjects with diabetes of long duration have shown thickening of the basement membrane of the choriocapillaries walls, luminal narrowing, dropout 
of the choriocapillaries, and thickening of the Bruch membrane, factors that may predispose to the development of AMD. ${ }^{21}$ However, there is poor evidence for a common pathogenetic pathway since data from epidemiological studies is inconsistent .

There is limited population-based evidence supporting a clear association between diabetes and AMD. In the Beaver Dam Eye Study, diabetes was not associated with early AMD. ${ }^{4}$ However, in persons older than 75 years, those with diabetes had a higher frequency of neovascular AMD (9.4\%) than those without diabetes $(4.7 \%)$ but both groups had similar frequencies of geographic atrophy. The relative risk of neovascular AMD in diabetic men over 75 years of age was 10.2 (95\% CI: 2.4-43.7); for women it was 1.1 (95\% CI: 0.4-3.0). The authors noted that they were unable to explain the observed relationship between neovascular AMD and diabetes in older men but not women and suggested that this might be the result of chance. ${ }^{4}$ In this context it is worth noting that amongst women who participated in the Women's Health Initiative Sight Exam Ancillary study, a history of diabetes was associated with a 2.5-fold increased risk for neovascular AMD but not associated with either early AMD or GA. ${ }^{3}$

In contrary, the Blue Mountains Eye Study reported that diabetes was significantly associated with the prevalence of geographic atrophy (OR: 4.0; 95\% CI, 1.6-10.3), but no association was found either for neovascular AMD (OR: 1.2; 95\% CI, 0.4-3.5) or early AMD (OR: $1.0: 95 \% \mathrm{CI}, 0.5-1.8) .{ }^{5}$ In the five and 10 year incidence studies of the same cohort, diabetes was also related to an increased risk of incident geographic atrophy (RR: 8.3 and 3.9 respectively), but not to neovascular AMD. ${ }^{9}$ In a cohort study of a black population in Barbados, a 2.7-fold association of diabetes history with incident AMD was reported; however, subtype analysis of AMD was not performed. ${ }^{6}$ Diabetes history was not associated with early AMD in this study either.

Possible associations between diabetes and AMD have been identified by the AREDS (Age-related Eye Disease Study), a randomized, prospective, clinical trial. A history of diabetes was associated with increased risk for incident neovascular AMD (OR: 1.88) and not for GA in persons at risk of developing advanced AMD in one eye. ${ }^{7}$

Dietary glycemic index (dGI) has been linked to AMD. High dGI was found on a cross sectional analysis of the AREDS cohort to be associated with an increased risk of early and late AMD in non-diabetic subjects. ${ }^{22}$ In another report of the same study, high dGI was reported to be associated with increased risk of AMD progression in persons with early stages of the disease. ${ }^{23}$ 
The dGI is a weighted average of the glycemic index of foods in the diet. High dGI is an indicator of poor quality dietary carbohydrate intake and has been implicated in the development of diabetes and cardiovascular disease. It may increase the risk of AMD through several common etiologic factors of diabetes and cardiovascular disease, including the formation of advanced glycation end products and increases in oxidative stress, inflammation, and hyperlipidemia.

A number of studies have suggested an association between AMD and history of cardiovascular disease. Since diabetes is a major risk factor for cardiovascular disease, the association of diabetes with AMD could be mediated by the presence of cardiovascular disease. However, in the Eureye study the association of diabetes with neovascular AMD was independent of a history of cardiovascular disease. After adjustment, cardiovascular disease was not significantly associated with neovascular AMD (OR: 1.48; 95\% CI: 0.83-2.64; $\mathrm{p}=0.2$ ).

The EUREYE study is population based and because the participants were aged 65 or older there were more cases of late AMD than previous epidemiological studies, thus giving it additional power to detect associations with risk factors. However the study also has several limitations. Our results were based only on self-reported history of diabetes and participants may have incorrectly reported their diabetes status. However, self-reports of certain chronic conditions like diabetes are consider reasonably accurate and prevalence estimates of selfreported diabetes has been found to be similar to those from clinical and laboratory evaluations. ${ }^{24,25}$ In our study, people with a self reported history of diabetes were four times more likely to have retinal haemorrhages on fundus photography and 23 times more likely to have bilateral haemorrhages lending strength to the the validity of the self reported measure. In addition, we have no reason to believe that any misreporting was related to AMD status. We cannot exclude the possibility that people with AMD were more likely to be attending an ophthalmologist and therefore identified with diabetes earlier than the controls persons. However, the majority of people (69\%) with NV- AMD in our study did not report a history of AMD.

In conclusion, the EUREYE study results support a positive association between history of diabetes with neovascular AMD but not with geographic atrophy or early AMD. The hypothesis that diabetes mellitus is associated only with neovascular AMD may suggest different pathogenesis of the two advanced forms of the disease and needs to be further evaluated. 


\section{References}

1. Klaver CC, Wolfs RC, Vingerling JR, et al. Age-specific prevalence and causes of blindness and visual impairment in an older population: the Rotterdam study. Arch Ophthalmol. 1998;116:653-658.

2. Snow KK, Seddon JM. Do age-related macular degeneration and cardiovascular disease share common antecedents? Ophthalmic Epidemiol 1999;6;124-143.

3. Klein R, Klein BE, Jeensen SC. The relationship of cardiovascular disease and its risk factors to the 5-year incidence of age-related maculopathy. The Beaver Dam Eye study. Ophthalmology 1997;104:1804-1812.

4. Klein R, Klein BE, Moss SE. Diabetes, hyperglycemia, and age-related maculopathy. The Beaver Dam Eye Study. Ophthalmology 1992;99:1527-1534.

5. Mitchell P, Wang JJ. Diabetes, fasting blood glucose and age-related maculopathy. The Blue Mountains Eye Study. Aust NZ J Ophthalmol 1999;27:197-199.

6. Leske MC, Wu SY, Hennis A, et al. Nine-year incidence of age-related macular degeneration in the Barbados Eye Studies. Ophthalmology 2006;113:29-35.

7. Age-Related Eye Disease Study Research Group. Risk factors for the incidence of advanced age-related macular degeneration in the Age-Related Eye Disease Study. AREDS report No 19. Ophthalmology 2005;112:533-539.

8. Smith W, Assink J, Klein R, et al. Risk factors for age-related macular degeneration. Pooled findings from three continents. Ophthalmology 2001;108:697-704.

9. Tomany S, Wang JJ, van Leeuwen R, et al. Risk factors for incident age-related macular degeneration. Pooled findings from 3 continents. Ophthalmology 2004;111:1280-1287.

10. Goldberg J, Flowerdew G, Smith E, Brody JA, Tso MO. Factors associated with age-related macular degeneration. An analysis of data from the first National Health and Nutritional Examination Survey. Am J Epidemiol 1988;128:700-710. 
11. Eye Disease Case Control Study Group. Risk factors for neovascular age-related macular degeneration. Arch Ophthalmol 1992;110:104-109.

12. Hyman L, Schachat AP, Ha Q, Leske MC. Hypertension, cardiovascular disease, and agerelated macular degeneration. Age-related Macular Degeneration Risk Factors Study Group. Arch Ophthalmol 2000;118:351-358.

13. Fraser-Bell S, Wu J. Klein R, et al. Cardiovascular risk factors and age-related macular degeneration: The Los Angeles Latino Eye study. Am J Ophthalmol 2008;145:308-316.

14. Delcourt C, Michel F, Colvez A, Lacroux A, Delage M, Vernet MH. Association of cardiovascular disease and its risk factors with age-related macular degeneration: the POLA study. Ophthalmic Epidemiol 2001;8:237-249.

15. Klein R, Meuer SM, Knudtson MD, Iyengar SK, Klein BEK. The epidemiology of retinal reticular drusen. Am J Ophthalmol 2008;145:317-326.

16. Augood C, Fletcher AE, Bentham G, et al. Methods for a population-based study of the prevalence of and risk factors for age-related maculopathy and macular degeneration in elderly European populations: the EUREYE study. Ophthalmic Epidemiol 2004;2:117-129.

17. Weinbrenner T, Vioque J, Barber X, Asensio L. Estimation of height and body mass index from demi-span in elderly individuals. Gerontology 2006;52:275-81.

18. The International ARM Epidemiological Study Group. An international classification and grading system for age-related maculopathy and age-related macular degeneration. Surv Ophthalmol 1995;39:367-374.

19. Van Leeuwen R, Chakravarty U, Vingerling H, et al. Grading of age-related maculopathy for epidemiological studies: Is digital imaging as good as $35 \mathrm{~mm}$ film? Ophthalmology 2003;110:1540-1544.

20. Augood CA, Vingerling JR, de Jong PT, et al. Prevalence of age-related maculopathy in older Europeans: the European Eye Study (EUREYE). Arch Ophthalmol 2006;124:529-535.

21. Hidayat AA, Fine BS. Diabetic choroidopathy light and electron microscopic observations of seven cases. Ophthalmology 1985;92:512-522.

22. Chiu C-J, Milton RC, Gensler G, Taylor A. Association between dietary glycemic index and age-related macular degeneration in non-diabetic participants in the Age-Related Eye Disease Study. Am J Clin Nutr 2007;86:180-188. 
23. Chiu C-j, Milton RC, Klein R, Gensler G, Taylor A. Dietary carbohydrate and the progression of age-related macular degeneration: a prospective study from the Age-Related Eye Disease Study. Am J Clin Nutr 2007;86:1210-1218.

24. Martin LM, Leff M, Calonge N, Garrett C, Nelson DE. Validation of self-reported chronic conditions and health services in a managed care population. Am J Prev Med 2000;18:215218.

25. Zhang X, Gregg EW; Cheng YJ, et al. Diabetes Mellitus and Visual Impairment National Health and Nutrition Examination Survey, 1999-2004. Arch Ophthalmol 2008;126:14211427.

Competing Interest: None declared 